\title{
The spectrum of the SB2 HD 64491 and the problem of metal abundances in $\lambda$ Boo stars ${ }^{\star}$
}

\author{
R. Faraggiana ${ }^{1}$ and M. Gerbaldi ${ }^{2,3}$ \\ 1 Dipartimento di Astronomia, Università degli Studi di Trieste, Via G.B.Tiepolo 11, 34131 Trieste, Italy \\ e-mail: faraggiana@ts.astro.it \\ 2 Institut d'Astrophysique, 98bis Bld Arago, 75014 Paris, France \\ e-mail: gerbaldi@iap.fr \\ ${ }^{3}$ Université de Paris Sud XI
}

Received 10 July 2002 / Accepted 10 October 2002

\begin{abstract}
The spectrum of the classical $\lambda$ Boo star HD 64491 reveals that the object is a double-lined spectroscopic binary whose components have different $v \sin i$ values. From such a tangled spectrum, we show that large metal underabundances can be derived with the hypothesis that the spectrum is formed by a single object, while the abundance peculiarities are drastically reduced when the duplicity is taken into account.
\end{abstract}

Key words. stars: atmospheres - stars: binaries: spectroscopic - stars: chemically peculiar

\section{Introduction}

The star HD 64491 belongs to the class of $\lambda$ Boo stars: this is a class of chemically peculiar (CP) stars characterized by underabundances of most elements heavier than He.

The abundance anomalies of $\lambda$ Boo stars are not homogeneous; even the definition of this group varies from author to author (see Faraggiana \& Gerbaldi 1998); consequently there are significant differences in the object catalogues proposed by different authors.

The metal deficiencies cannot be interpreted as an age effect since the majority of these stars appear to lie on or near the main sequence when their $T_{\text {eff }}$ values are derived from the photometric colour indices. Thus, the observed abundance anomalies must be confined to the atmospheric layers, which should have been modified by some photospheric mechanism or external agent.

Several mechanisms may have modified the atmospheric composition of these stars; the main agents explored up to now have been: diffusion, accretion and two stars merging (Charbonneau 1983; Venn \& Lambert 1990; Andrievsky 1997; Turcotte 2002). Each of these theories explains some, but not all, of the complicated and heterogeneous abundance patterns emerging from the spectral analyses; this intriguing situation explains the great efforts made in recent years to enlarge the number of known $\lambda$ Boo stars and to analyze their spectra.

\footnotetext{
Send offprint requests to: R. Faraggiana, e-mail: faraggiana@ts.astro.it

* Based on observations collected at the Observatoire du Pic du Midi (France).
}

However, almost all the abundance analyses made up to now are based on the hypothesis that $\lambda$ Boo stars are peculiar single objects, whose spectra are characterized by faint and, in most cases, broad metal lines. The only exceptions are the two SB2 stars, HD 84948 and HD 171948 (Paunzen et al. 1998a), but, at least for the former, the parameters and the abundances need to be revised (see Faraggiana et al. 2001a).

A number of $\lambda$ Boo stars have been found to be composed of two stars separated by less than 1 arcsec by Hipparcos and/or speckle interferometry (Faraggiana \& Bonifacio 1999). The suspicion that other binaries may have been misclassified as $\lambda$ Boo stars prompted Faraggiana et al. (2001a) to look for binaries that have too small an angular separation to be detected by direct imaging. These authors studied the spectra of some already-known binaries classified as $\lambda$ Boo in order to define criteria that make it possible to distinguish composite spectra; by applying these criteria, they demonstrated that HD 111786 is in reality a multiple system and (Faraggiana et al. 2001b) that HD 174005 is a spectroscopic binary whose spectrum is affected by that of the companion.

In the present paper we examine the spectrum of another classical $\lambda$ Boo star, HD 64491; it appears to be severely contaminated by that of a companion star, i.e. the flux collected by a spectrograph is the result of the combined fluxes of two dwarf stars having different rotational velocities and similar parameters. The computations demonstrate that the combination of two moderately underabundant spectra produce a composite spectrum that mimics that of a single star with large metal underabundances. 


\section{Optical and UV data}

HD 64491 is classified as a $\lambda$ Boo star according to Abt \& Morrell (1995) and Gray (1997); the latter author classified this star as kA3hF0mA3 $\lambda$ Boo PHL (Peculiar Hydrogen Lines).

HD 64491 has been named DD Lyn in the 75th name-list of variable stars (Kazarovets et al. 2000) after the measurement of pulsation by Paunzen et al. (1998b). Since then this star has been considered a $\delta$ Sct star and belongs to the catalogue of $\delta$ Sct stars by Rodriguez et al. (2000), with a period of $71 \mathrm{mn}$ and an amplitude of $0.009 \mathrm{mag}$ in the Strömgren $b$-filter.

Kamp et al. (2001) cited HD 64491 as a spectroscopic binary with an extremely broad-lined primary and a narrowlined secondary. However, neither these authors nor Kamp et al. (2002) took into account the contamination by the companion in deriving the atmospheric parameters on which they based their analysis of the abundances of $\mathrm{S}, \mathrm{N}$ and $\mathrm{O}, \mathrm{Ca}$. The variation in the radial velocity due to the orbital motion was observed by Iliev et al. (2001). Meanwhile, no sign of suspected duplicity was detected by the Hipparcos experiment, nor any sign of photometric variability. A speckle observation (Marchetti et al. 2001) gave a negative result for a binary separation higher than 124 mas.

The value of $v \sin i$ is $15 \mathrm{~km} \mathrm{~s}^{-1}$ according to Abt \& Morrell (1995); the value of $75 \mathrm{~km} \mathrm{~s}^{-1}$ given by Uesugi \& Fukuda (1982) and coming from Palmer et al. (1968) should be considered an upper limit, since it is based on spectra taken with a spectrograph having a projected slit width of $75 \mathrm{~km} \mathrm{~s}^{-1}$. In 2001, Kamp et al. used a much higher value, $v \sin i=170 \mathrm{~km} \mathrm{~s}^{-1}$ for their analysis of $\mathrm{S}$ and $\mathrm{N}$ abundances, but this value was obtained from the Paschen lines, which, being mainly broadened by the linear Stark effect, are not suitable for $v \sin i$ measures. In 2002, Kamp et al. measured different values of the projected rotational velocity: $v \sin i=25 \mathrm{~km} \mathrm{~s}^{-1}$ from the OI triplet and $60 \mathrm{~km} \mathrm{~s}^{-1}$ from the Ca II $\mathrm{K}$ line.

In the UV, the star has not been observed by IUE; the only available UV observations are the TD1 fluxes (Thompson et al. 1978 , in the revised edition available at CDS), which will be discussed in Sect. 4.2.

\section{Spectroscopic observations}

We collected optical spectra of HD 64491 at the MUSICOS echelle spectrograph (Resolution $=38000$ ) mounted on the $2.2 \mathrm{~m}$ TBL telescope of the Observatoire du Pic du Midi (France); the range covered by the chosen spectrograph setting is $515-889 \mathrm{~nm}$ and the average $S / N$ is 200 . The $S / N$ diminishes drastically toward the redder part of the spectrum due to the efficiency of the spectrograph in this domain, where, moreover, the orders do not overlap, preventing us from using this region in the present study.

Relevant information on the spectra are given in Table 1.

The photometric and wavelength reduction have been made with the ESpRIT code developed by Donati et al. (1997) and made available at the telescope site. Further spectral analysis has been performed by using the ESO MIDAS package.
Table 1. The observations: range $515-889 \mathrm{~nm}$.

\begin{tabular}{crc}
\hline \hline JD & date & exp. time (min) \\
\hline 2451621.4097 & Mar. 17, 2000 & 60 \\
2452307.4618 & Feb. 1, 2002 & 120 \\
2452310.5111 & Feb. 4, 2002 & 120 \\
2452314.4035 & Feb. 8, 2002 & 120 \\
2452316.4194 & Feb. 11, 2002 & 120 \\
\hline
\end{tabular}

\section{Analysis of the spectra}

\subsection{Variable features in the spectra: $\delta$ Sct versus duplicity effect}

We applied the criteria defined by Faraggiana et al. (2001a) to investigate the duplicity of HD 64491. A qualitative inspection of the spectra shows that most of the metal lines are quite narrow ( $v \sin i$ about $20 \mathrm{~km} \mathrm{~s}^{-1}$ ); on the contrary, the triplet at $777.2-777.4 \mathrm{~nm}$ of O I has a broad profile at the top of the feature, near the continuum, a peculiarity that is not expected since this feature is not blended with other lines in A-F type stars; moreover, the relative intensity and the line profiles of the three components change from night to night (Fig. 1). Also, the $\mathrm{Na}$ I doublet has a narrow plus broad profile similar to that of the above feature, in contrast with other weak metal lines. The core of the $\mathrm{H}_{\alpha}$ profile shows a slightly variable shape from night to night (Fig. 2). A slight variability in the line depth of some metal lines, mostly from ionized species, is also detected (Fig. 3).

We may ask ourselves whether these variations are due to the $\delta$ Sct properties of this star. It is known that the $\delta$ Sct pulsations produce variations in the line profiles: see, for example, the observations of the star $\theta$ Tuc plotted in Fig. 4 of the paper by De Mey et al. (1998). The integration time of our spectra (see Table 1) is longer than the pulsation period of $71 \mathrm{mn}$ measured by Paunzen et al. (1998b). The MUSICOS spectrograph covers a large spectral domain in one frame but does not permit a short exposure time. Nevertheless, it may be argued that small variations, due to $\delta$ Sct pulsation, are present in our spectra; the entity of a profile distortion in a pulsating star is detailed in the already-cited Fig. 4 of De Mey et al.'s (1998) paper, showing the profile variations of a metallic line on three different nights.

We conclude that, if part of the the variations that we observed in the metallic lines (Fig. 3) is due to the $\delta$ Sct pulsations, binarity is also responsible for these variations, because the modifications in the OI triplet profile from one night to the other (Fig. 1) cannot be attributed only to the pulsation phenomenon. A fundamental point in favour of the duplicity of this object is that the pulsation phenomenon cannot produce an inversion of the relative intensity of the lines of the OI triplet.

We have measured, on the spectra of Table 1, the position of the centroid of some metallic lines in order to derive the radial velocity. Figure 4 displays the radial velocity data obtained by Iliev et al. (2001) and by our measurements. We note that the behaviour of all these measurements reflects the orbital motion. These data are still too scarce to compute the period of the orbital motion. The non-smoothness of the data may reflect the fact that the radial velocity measured is a combination 


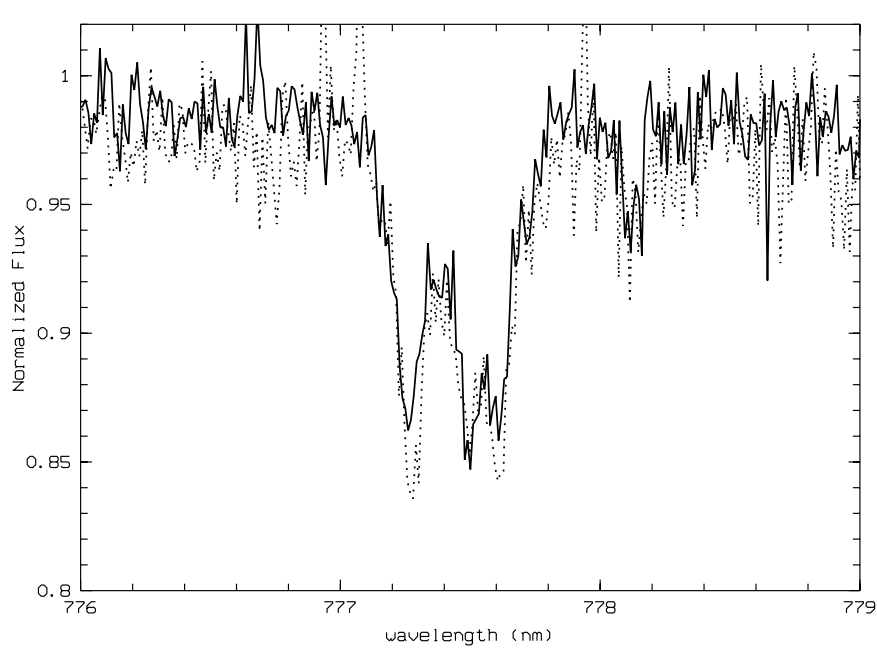

Fig. 1. The O I triplet in spectra taken on February 1 (thick line) and 4 (dotted), 2002.

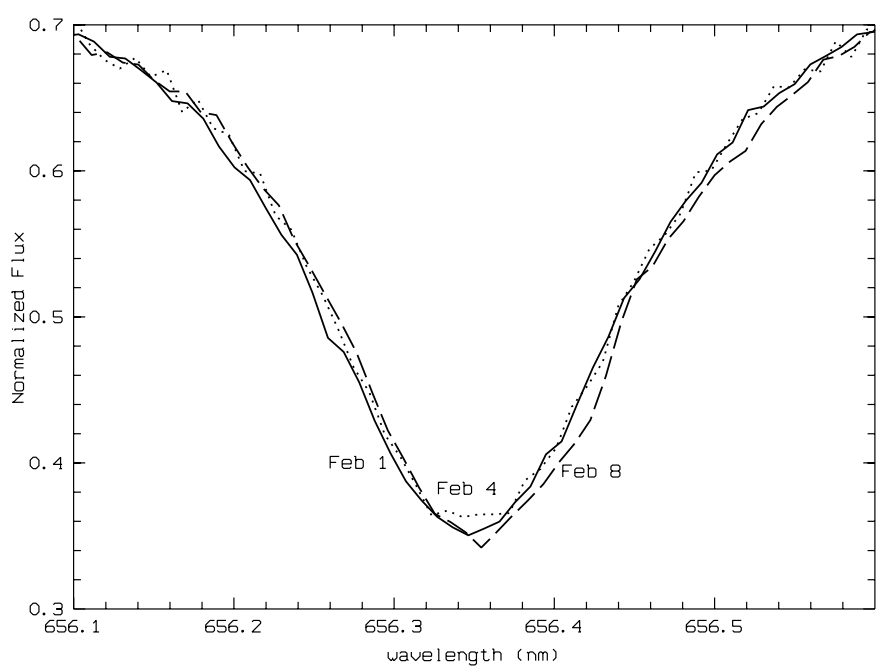

Fig. 2. The observed $\mathrm{H}_{\alpha}$ core on spectra taken on February 1 (thick), 4 (dotted) and 8 (dashed), 2002.

of the orbital motion and of the pulsations. It is also due to the difficulty in measuring the precise radial velocity because the spectrum of this binary system consists of blends of narrow and broad lines with a relative motion; this prevents the accurate measurement of the radial velocity from non-blended lines.

So HD 64491 displays the properties of a composite object; we quantify the composite spectrum characteristics in the following sections.

\subsection{Atmospheric parameters and evolutionary stage of the system}

In spite of the signs of duplicity found in the spectra, we start by comparing our observations with a synthetic spectrum computed with the atmospheric parameters derived from photometric colour indices of HD 64491 considered as an ideal single object, an approach previously used for this and other $\lambda$ Boo stars.

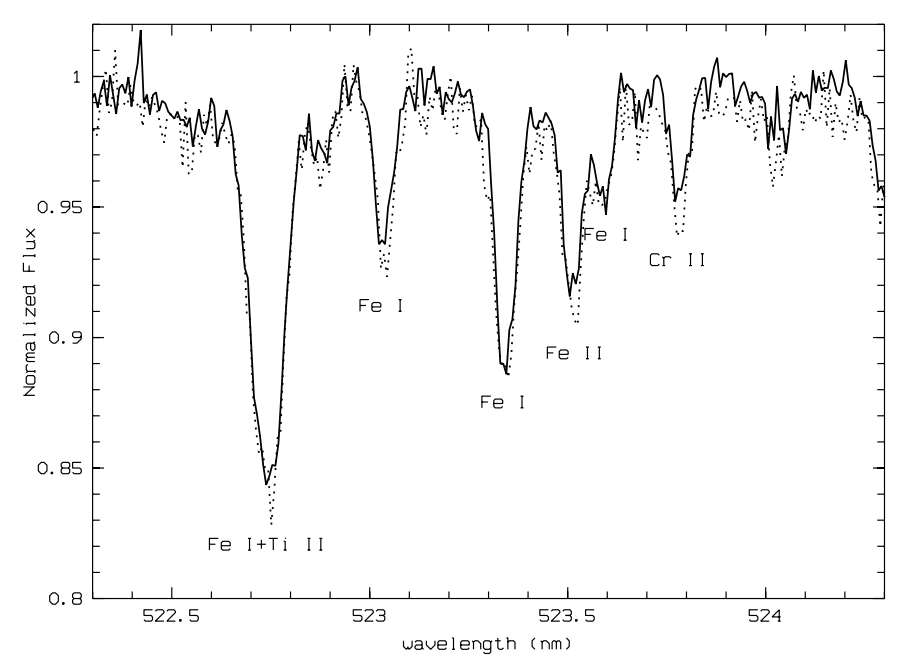

Fig. 3. Examples of metal lines in spectra taken on February 1 (thick) and 4 (dotted), 2002.

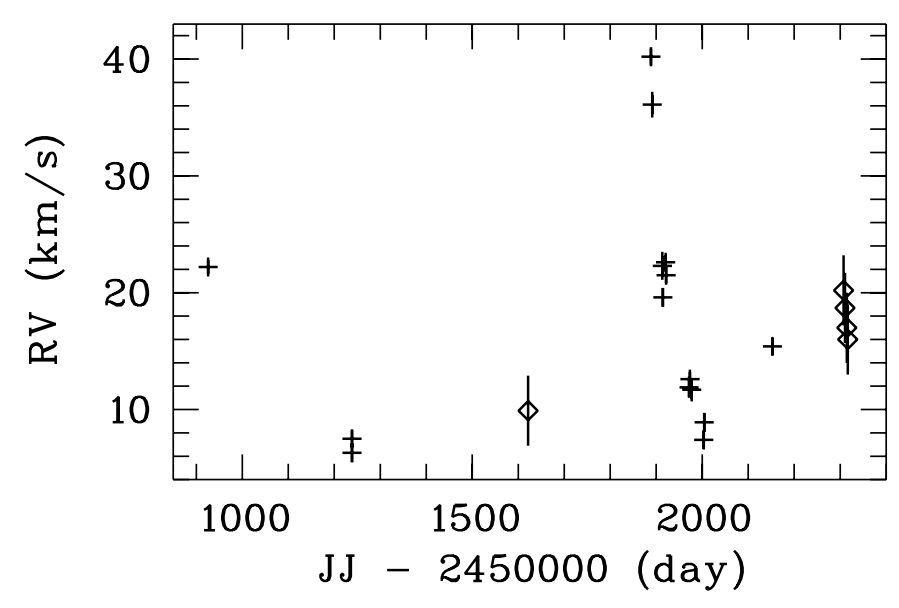

Fig. 4. The radial velocities given by Iliev et a. (2001) (cross) and measured from our spectra (diamond) according to the JJ day of the observation. The error bars on the radial velocities are given.

We stress that the procedure used to determine $T_{\mathrm{eff}}$ and $\log g$ from photometric colour indices is physically correct only if the star is a single object. This approach is used here to obtain a rough approximation of the parameters of the stars and to derive the constraints on the likely luminosity values of the two binary system components; these values cannot be considered the true atmospheric parameters for an elemental abundance analysis.

We determined the atmospheric parameters from $u v b y \beta$ and Geneva photometries by using the Moon \& Dworetsky (1985) and Künzli et al. (1997) calibrations of the $u v b y \beta$ and Geneva photometric colour indices. The color excess $E(b-y)$ was computed with the Moon (1985) programme. Its value is: $E(b-y)=-0.010$, so we adopted the value 0.0 for the redenning.

The parameters so derived are: $T_{\text {eff }}=7120 \mathrm{~K}, \log g=4.08$ from $u v b y \beta$ photometry and $T_{\text {eff }}=7304 \mathrm{~K}, \log g=4.56$, $[\mathrm{M} / \mathrm{H}]=-0.67$ from the Geneva one.

Concerning the error in the determination of $T_{\text {eff }}$, we adopt $150 \mathrm{~K}$ as an upper limit, for both effective temperature 


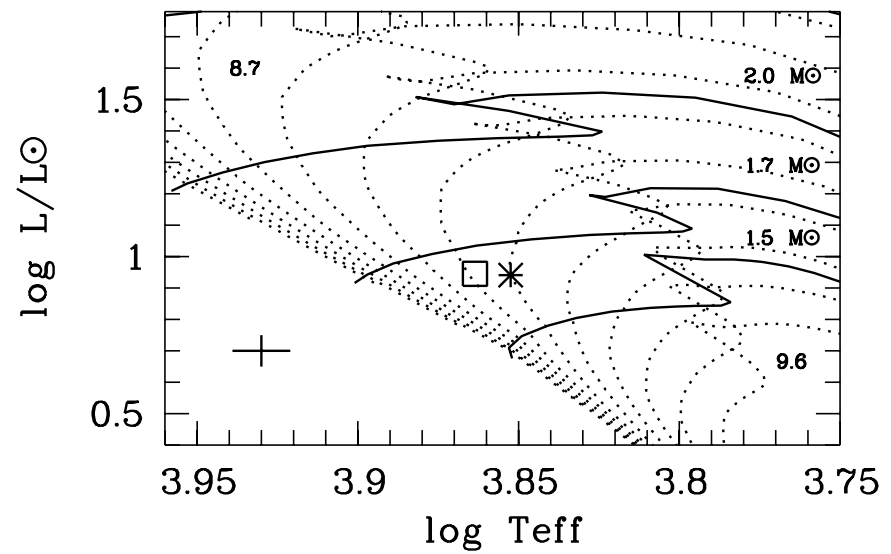

Fig. 5. The star HD 64491 is plotted on the HR diagram with isochrones from Schaller et al. (1992) (dashed lines) and evolutionary paths (continuous line) from Meynet et al. (1993). The "square" corresponds to the $T_{\text {eff }}$ value from the Geneva photometry calibration and the "asterisk" from the MD calibration. The ages (yr) on the isochrones are given in logarithmic scale at each 0.1 interval; only two values of the ages are indicated.

calibrations. This value comes from our experience using these calibrations of $T_{\text {eff }}$; discussion of the error in the $T_{\text {eff }}$ determination can be found, for example, in Gerbaldi et al. (1999), and in Gerbaldi et al. (2001).

The variability due to the pulsations affects the atmospheric parameters. As photometric variations have been measured in only one filter, the $b$-band of the Strömgren photometry, it is impossible to quantify the impact of these pulsations on the values of $T_{\text {eff }}$ and $\log g$. As an example, we refer to the paper by Bessell (1969), where this effect is estimated for pulsating stars: for the star $\delta$ Del, which has a variation in $B$ mag of $0.05 \mathrm{mag}$, the variation on $T_{\text {eff }}$ is $180 \mathrm{~K}$. The amplitude of the variation of HD 64491 being much lower than that of $\delta$ Del, we estimate that the resulting error in $T_{\text {eff }}$ is less than $180 \mathrm{~K}$.

The visual absolute magnitude is computed from the parallax given in the Hipparcos Main Catalogue (ESA 1997) $\pi=$ $16.55 \pm 0.92$ mas; the value of $M_{\text {bol }}$ is computed by applying the bolometric correction taken from Bessell et al. (1998), assuming for the sun the value $M_{\mathrm{bol} \odot}=4.75$. The stellar $M_{\mathrm{bol}}$ is converted into $\log L / L_{\odot}$ to place the star on the HR diagram of Fig. 5, where the evolutionary tracks are taken from Schaller et al. (1992) and the isochrones from Meynet et al. (1993).

The errors in $L / L_{\odot}$ arise from the computation of $M_{\mathrm{bol}}$, that is mainly from those in $M_{V}$ (we do not take into account any error on the bolometric correction).

As $M_{V}=V+5+5 \log (\pi)-A v$, the error in $M_{V}$ can be expressed as:

$\sigma\left(M_{V}\right)=\left((\sigma(V))^{2}+(2.17 \sigma(\pi) / \pi)^{2}+(\sigma(A v))^{2}\right)^{0.5}$.

As a rule of thumb, a value of 0.01 is adopted for $\sigma(V)$ and 0.04 for $\sigma(A v))$ taking into consideration that this object is at about $60 \mathrm{pc}$ and that we have neglected the interstellar absorption in the direction of the star.

The value of $\sigma\left(\log \left(L / L_{\odot}\right)\right)$ is equal to $\sigma(M v) / 2.5$, that is 0.05 .

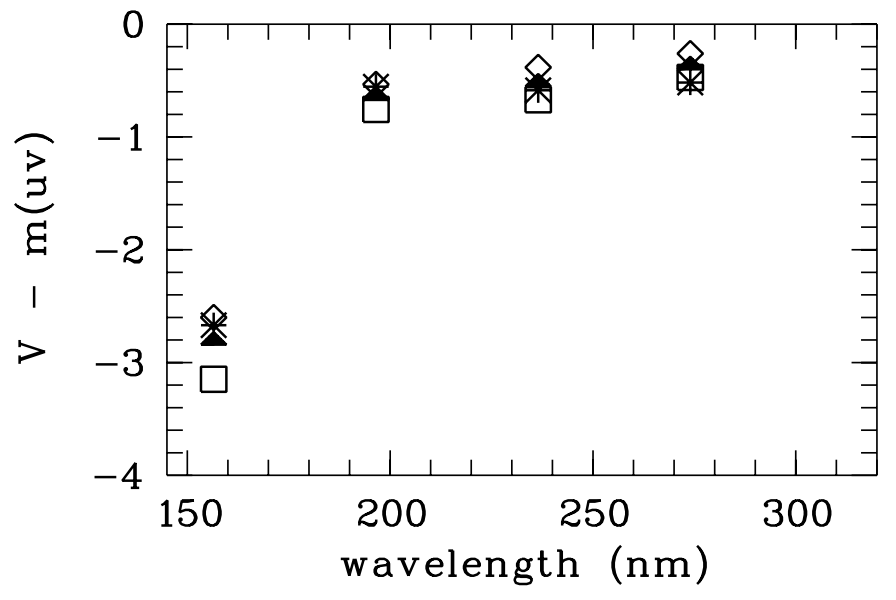

Fig. 6. The UV colour indices: $V-m(u v)$ of HD 64491 measured by the S2/68 experiment on board the TD1 satellite are compared with synthetic indices computed from Kurucz fluxes integrated over the same wavelength bands at 1565, 1965, 2365 and 2740 A. The observations are represented by an "asterisk", $[\mathrm{M} / \mathrm{H}]=-1.0$ by a "square", -1.5 by a "filled triangle" and -2.0 by a "diamond". The errors in the colour indices are respectivly for each spectral band: $0.1,0.04,0.01$ and 0.01 .

The UV flux observations by the S2/68 experiment on board the TD1 satellite have been compared with the Kurucz computed fluxes integrated over the band width of each TD1 channel. A metal underabundance $[\mathrm{M} / \mathrm{H}]$ in the range -1.5 to -2 . is required to fit the observed with the computed behaviour of the UV spectrum (Fig. 6).

By combining all this informations, we choose the following parameters to compute a synthetic spectrum by using the Kurucz (1993) model atmosphere and his SYNTHE program: $T_{\text {eff }}=7250 \mathrm{~K}, \log g=4.0$ and $[\mathrm{M} / \mathrm{H}]=-1.0$ for all but CNOS elements, for which solar abundances have been adopted to better reproduce the expected behaviour of $\lambda$ Boo stars; the value of $2 \mathrm{~km} \mathrm{~s}^{-1}$ is used for the microturbulence velocity, and the chosen $v \sin i=20 \mathrm{~km} \mathrm{~s}^{-1}$ is obtained from the best fit between the observed and the computed metal line profiles.

\subsection{Comparison of the observed and computed spectrum}

The spectrum computed with the above parameters has been superimposed on that observed on March 17, 2000; the regions of the $\mathrm{Na}$ I doublet and of the $\mathrm{Mg}$ I triplet are displayed in Figs. 7 and 8.

The comparison clearly shows that a single spectrum cannot reproduce the observed profiles of strong lines, in particular those of the O I triplet and of the Na I doublet. The most convincing proof of the composite spectrum of HD 64491 is given by the shape of the $\mathrm{O}$ I triplet: the relative intensities of the three components are variable and, especially in the Feb. 1, 2001 spectrum, do not reflect the theoretical central depth ratios, according to which $777.2 \mathrm{~nm}$ should be the strongest component (Fig. 1). The companion star is responsible for the underlying broad feature, the single characteristic blend of O I 777.2777.4 observed in highly rotating stars. This is illustrated in Fig. 9, where the spectrum observed on March 17, 2000, is 


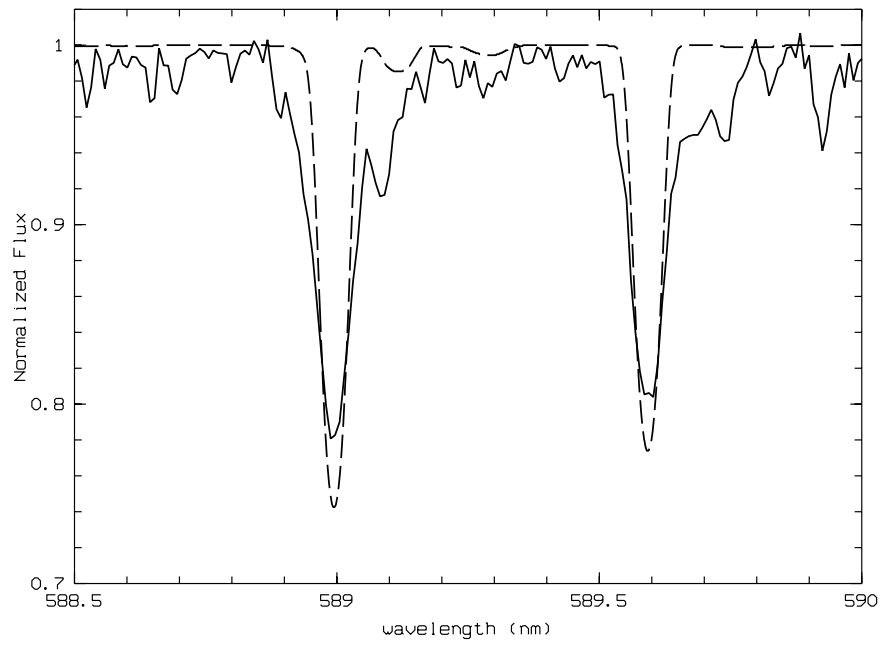

Fig. 7. The observed Na I doublet (thick) compared with the spectrum computed with $T_{\text {eff }}=7250, \log g=4.0$ and $[\mathrm{M} / \mathrm{H}]=-1.0$; $v \sin i=20 \mathrm{~km} \mathrm{~s}^{-1}$ (dotted).

plotted with the two computed spectra based on the same model atmosphere, but with $v$ sin $i$ values of 20 and $80 \mathrm{~km} \mathrm{~s}^{-1}$ respectively; the observed broad component appears to fill the upper part of the observed profile.

A similar signature of a broad-line component is easily detectable in the NaI 589.0-589.6 nm doublet, in the line profiles of the $\mathrm{Mg}$ I triplet 517.3-518.3 (Figs. 7 and 8) and in the strongest Fe I lines; a red-shift of the broad component with respect to the narrow one is also evident from these figures.

If we accept the hypothesis that the system is composed of two stars, these must have significantly different rotational velocities, probably due to the different inclination of their rotational axes; we estimate that the companion star has $v$ sin $i=$ $80 \mathrm{~km} \mathrm{~s}^{-1}$ and a differential radial velocity of $20 \mathrm{~km} \mathrm{~s}^{-1}$. An alternative hypothesis is that the broad component is a convolution of two sources each of lower $v \sin i$.

The alternative interpretation that the two line components with different width and position are generated in the atmosphere of a single star must be rejected since HD 64491 is a dwarf star which is expected to emit flux only from its photospheric layers.

\section{A combined spectrum and its effect on the apparent metallicity}

The synthetic spectra to be combined must be chosen in such a way that they take into account the position of the "combined" object in the HR diagram (Fig. 5). The two components cannot have very different magnitudes, otherwise only one spectrum would have been observed; this imposes a limit for selecting the $T_{\text {eff }}$ for the theoretical spectra. One of the stars is probably cooler and the other hotter than the $T_{\text {eff }}$ value of $7120-7320 \mathrm{~K}$ derived from the combined light, but according to the position of the "combined" object in Fig. 5 each of these $T_{\text {eff }}$ values cannot be higher than $7500 \mathrm{~K}$. We can easily notice, from this figure, that if we take two "ideal" stars with the same $T_{\text {eff }}=$ $7250 \mathrm{~K}$ and the same luminosity on the ZAMS, the combined

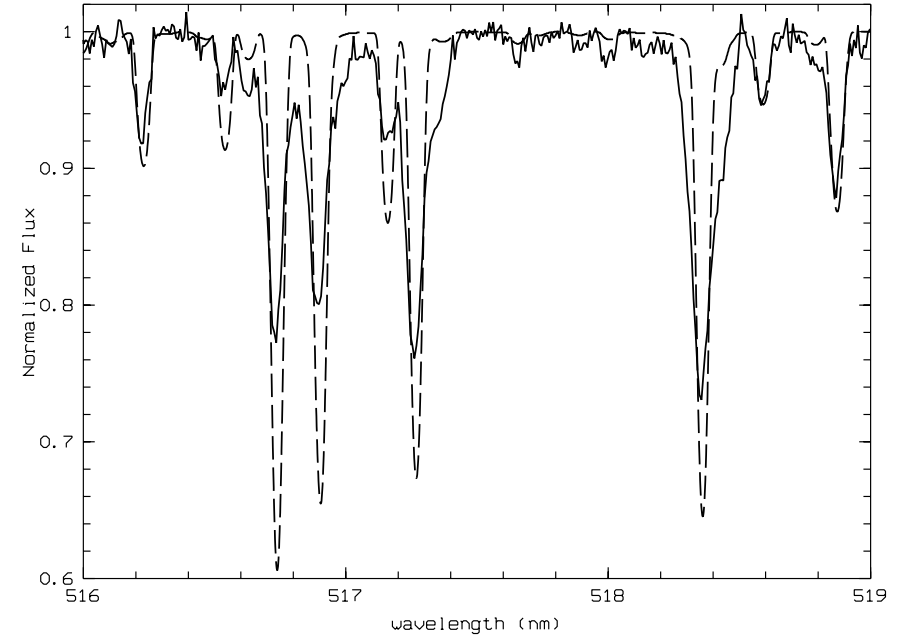

Fig. 8. The observed Mg I triplet (thick) compared with the spectrum computed with $T_{\text {eff }}=7250, \log g=4.0$ and $[\mathrm{M} / \mathrm{H}]=-1.0 ; v$ sin $i=$ $20 \mathrm{~km} \mathrm{~s}^{-1}$.

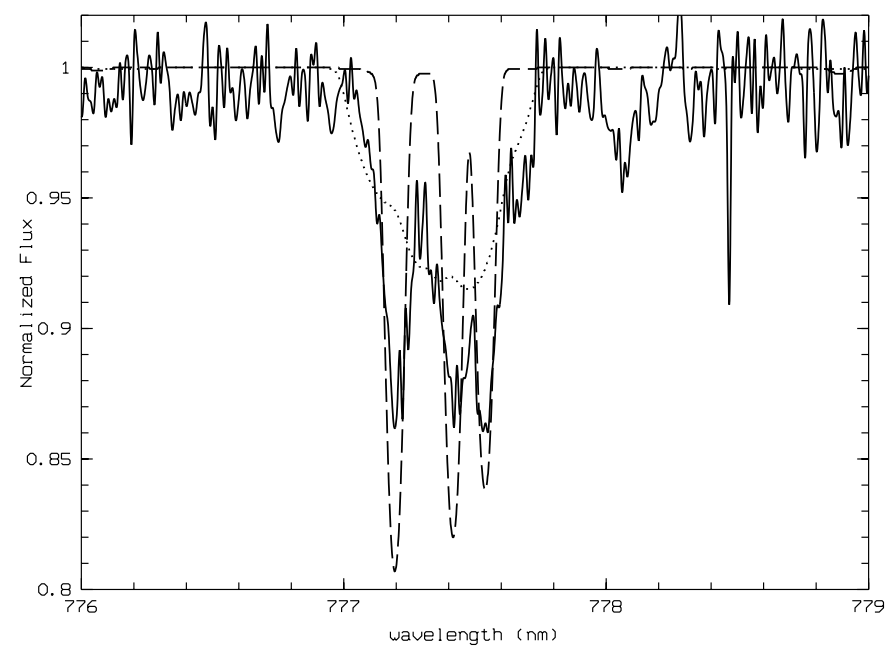

Fig. 9. The observed O I triplet (March 17, 2000) (thick) compared with two theoretical spectra computed with $v \sin i=20$ (dashed) and $80 \mathrm{~km} \mathrm{~s}^{-1}$ (dotted) and $T_{\text {eff }}=7250, \log g=4.0$ and $[\mathrm{M} / \mathrm{H}]=-1.0$ but solar CNOS abundances.

object has a luminosity 0.3 dex higher, and the resulting object is located in the HR diagramme very near to the observed one.

In order to better reproduce the observations, two spectra with the same model atmosphere having $T_{\text {eff }}=7250 \mathrm{~K}$, $\log g=4$. and $[\mathrm{M} / \mathrm{H}]=-0.5$, but with $v \sin i=20$ and $80 \mathrm{~km} \mathrm{~s}^{-1}$ have been computed and combined by assuming the same luminosity; a RV difference of $+20 \mathrm{~km} \mathrm{~s}^{-1}$ has been introduced for the second one for comparison with the higherquality observed spectrum, that obtained on March 17, 2000.

This combined spectrum is compared with the observed one in the region of the $\mathrm{Na} \mathrm{I}$ doublet and the $\mathrm{Mg}$ I triplet in Figs. 10 and 11; we have also plotted the spectrum computed from a single model having the same $T_{\text {eff }}$ and $\log g$, but metal abundances 1 dex lower, more precisely $[\mathrm{M} / \mathrm{H}]=-1.5$ for all but the $\mathrm{C}, \mathrm{N}, \mathrm{O}$ and $\mathrm{S}$ elements.

Consequently, the abundances derived from an analysis in which the star is considered as single will appear lower by 


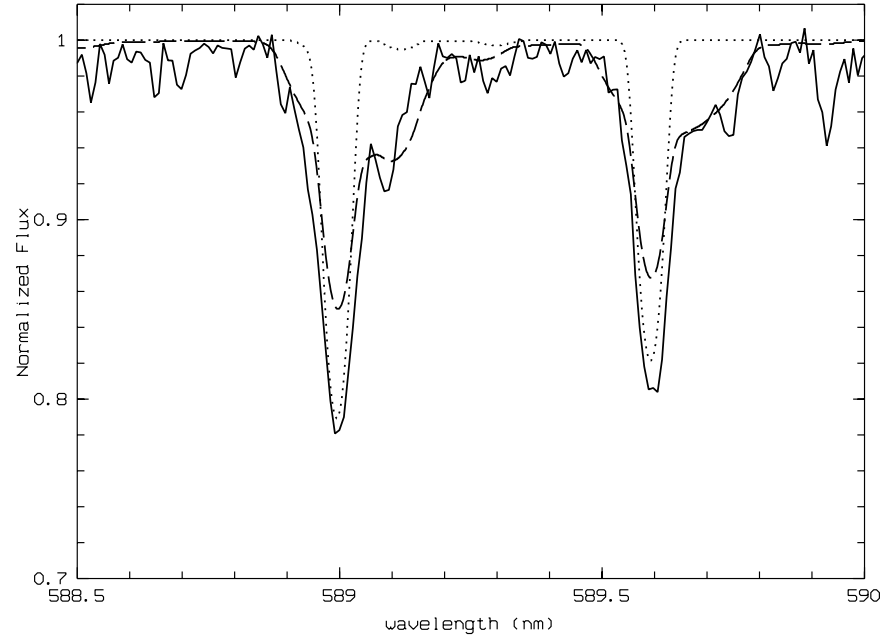

Fig. 10. The $\mathrm{Na}$ I doublet compared with a combined spectrum (dashed) from two spectra having both the same $T_{\text {eff }}$ and $\log g$ $(7250,4.0),[\mathrm{M} / \mathrm{H}]=-0.5$ and only different $v \sin i(20$ and $80 \mathrm{~km} \mathrm{~s}^{-1}$ ); the broad component has been shifted by $+20 \mathrm{~km} \mathrm{~s}^{-1}$. The dotted line refers to the single computed spectrum having with the same $T_{\text {eff }}$ and $\log g$ values, but $[\mathrm{M} / \mathrm{H}]=-1.5$ and $v \sin i=20 \mathrm{~km} \mathrm{~s}^{-1}$.

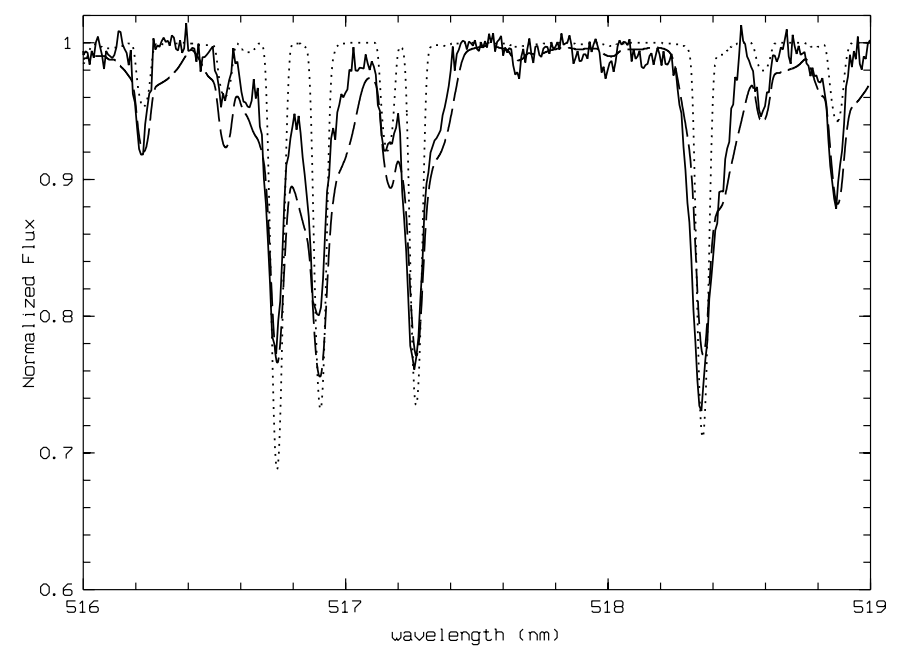

Fig. 11. The Mg I triplet compared with the same computed spectra as those in Fig. 10.

about 1 dex. We stress that the computed profiles of the NaI doublet and the MgI triplet are deeper in the single, more metaldeficient spectrum than in that obtained from the combination of the two less deficient spectra.

In Fig. 12, a region dominated by Fe lines, the observed spectrum is also compared with these two computed spectra. We note that some lines are fitted by the computed combination of spectra, while others are either too weak or too strong, thus indicating that the chosen atmospheric parameters are only a rough estimation of the true ones.

Figure 13 displays the observed O I triplet compared to a theoretical spectrum computed with the parameters used by Kamp et al. (2002) to derive the oxygen abundance. These authors give, in their Table 4, the abundance derived from the 777.2 triplet in LTE; this is the abundance we have introduced into the computations. We note the large discrepancy between

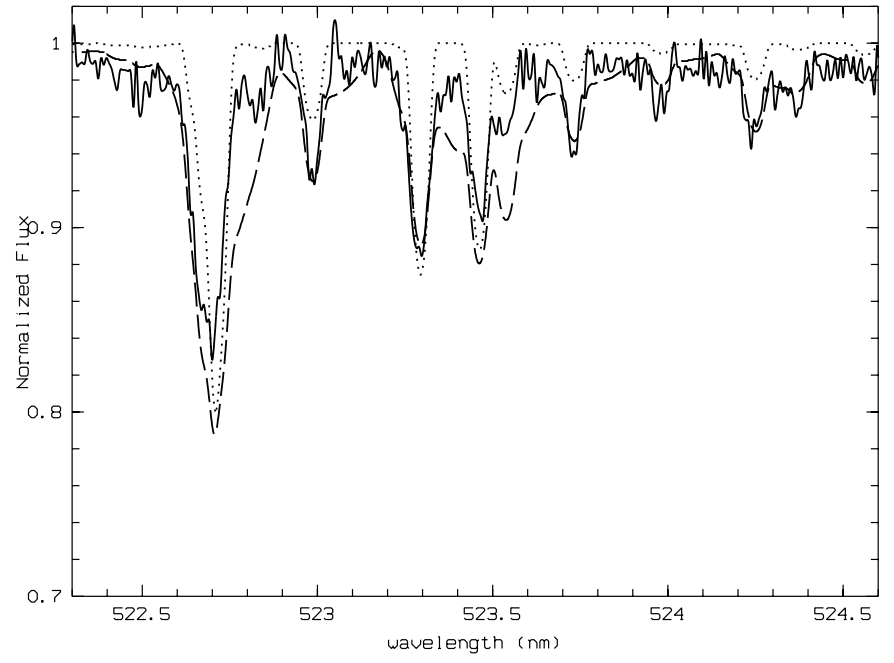

Fig. 12. Metal lines compared with the same computed spectra as those in Figs. 10 and 11.

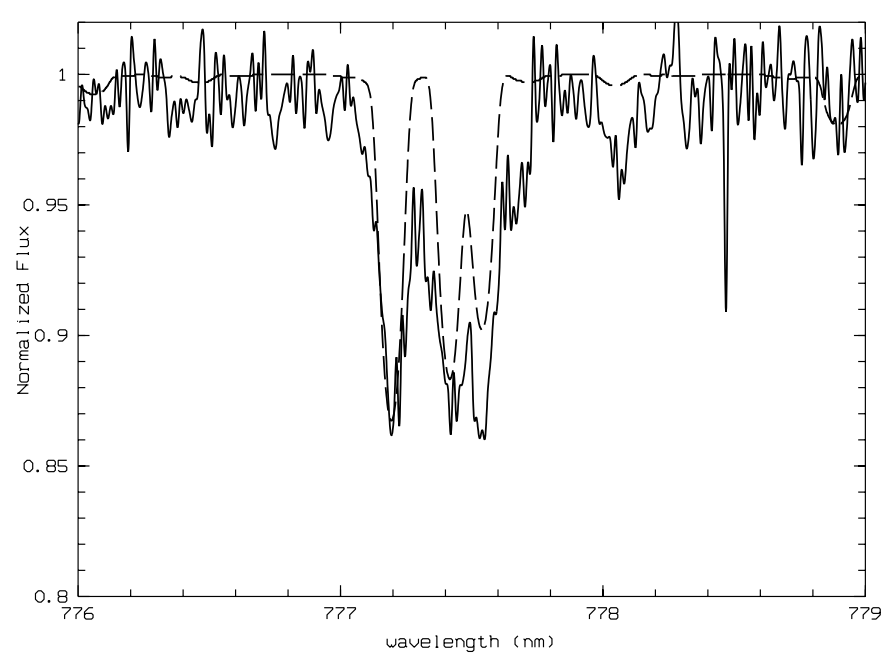

Fig. 13. The OI triplet observed on March 17, 2000 (thick) compared with the spectrum computed by adopting the model, the $v \sin i$ and the LTE oxygen abundance obtained by Kamp et al. (2002); $T_{\text {eff }}=7140 \mathrm{~K}$, $\log g=4.1$, microturbulence $=3 \mathrm{~km} \mathrm{~s}^{-1}$ and $v \sin i=25 \mathrm{~km} \mathrm{~s}^{-1}$.

the observed spectrum and the computed one, in the framework of a single object.

We note that our computations, partly illustrated in the figures given, show that the single spectrum computed with $[\mathrm{M} / \mathrm{H}]=-1.5$ does not reproduce the observed spectral line intensities either, and we stress that the combination of two spectra with different $v$ sin $i$ values and $[\mathrm{M} / \mathrm{H}]=-0.5$ may roughly mimic a single spectrum with $[\mathrm{M} / \mathrm{H}]=-1.5$, giving rise to an uncertainty of $1 \mathrm{dex}$ in the abundance determination of binaries formed of two stars having equal parameters, but different rotational velocities.

\section{Discussion}

The detailed inspection of the HD 64491 spectrum has revealed that this object is an SB2 star; in fact, the profiles of the strong lines appear to be due to two objects characterized by different $v \sin i$ values. 
The attempts to reproduce the observed spectrum by combining two computed spectra whose only known constraints are the total luminosity of the system and the average atmospheric parameters reveal that this task is anything but easy.

The mixture of spectra from two stars with similar, but not necessarily identical, parameters without any further knowledge except the distance of the system is tremendously difficult to handle accurately and univocally; in fact, the problem does not have one single analytical solution.

Very few abundance analyses of binary stars producing tangled spectra have been made up to now. The most thoroughly studied spectra of the double-lined spectroscopic binaries are those known as classical "composite" spectra, according to the definition by Griffin \& Griffin (1986), and are produced by double-lined spectroscopic binaries of comparable luminosity, but composed of a red giant or supergiant and a main-sequence star of A or B-type. These authors started a series of papers on this subject by splitting the composite spectrum of HR 6902 . They classified the two components by subtracting the spectrum of a single late-type giant used as reference star, i.e. by using a classical technique already applied successfully before the advent of large computing facilities (Wright 1954; Wright \& Lee 1959; Lee \& Wright 1960) to derive the spectrum of secondaries of Capella and $\zeta$ Aur stars. The Griffin project was initially devoted to determining precise masses of cool giants, which are otherwise difficult to obtain for single stars, and to studying the chromospheres of primaries of $\zeta$ Aur eclipsing binaries. The most recent extension of this program (Griffin 2002) concerns the spectrum of $o$ Leo, a binary composed of a late F-type giant $\left(T_{\text {eff }}=6100 \mathrm{~K}, \log g=3.25\right)$ and a dwarf Am $\left(T_{\text {eff }}=7600 \mathrm{~K}, \log g=4\right)$, whose spectra both have sharp lines ( $v$ sin $i=11$ and $8 \mathrm{~km} \mathrm{~s}^{-1}$, respectively). The successful separation of the components of this non-eclipsing binary is based on a series of 27 spectra covering the known orbital period, and analyzed by the FDBINARY package, a Fourier disentangling algorithm under development by Ilijc et al. (2001); it identifies the co-moving frames in a series of spectra. In order to make this method successful, the input spectra must uniformly cover the period phases, whose values are required as input parameters; moreover the determination of the intrinsic spectra requires the knowledge of the magnitude difference between the two component stars to derive their correct brightness ratio. Even in these favourable case the chemical analysis and the exact $v \sin i$ values are considered preliminary by this author, who calls for more sophisticated models.

The situation described above is far from being comparable with that of the $\lambda$ Boo stars of our program for which, on the basis of one or a few spectra, we may only hope to recognize the duplicity if the phase allows us to do so; with only a rough indication of radial velocity variations and of the orbital period, there is no hope of solving correctly and univocally the problem, and the abundances cannot have a physical meaning until these parameters are obtained more accurately. We can only stress that the correct determination of chemical abundances is crucial for the development of theories on the origin of this class of stars.

Lastly, we wish to point out that HD 64491 is a pulsating star of $\delta$ Sct type; the influence of this variability on the line profile shape and the possible variation in temperature with the short period $(P=0.049 \mathrm{~d})$ cannot be clarified with the spectra at our disposal; in fact, the necessary exposure times are of the same order as the period length. We can only mention that the abundances of $\delta$ Sct stars, obtained by assuming a constant $T_{\text {eff }}$ and based on spectra averaged over the whole variability period, indicate that the elements of the Fe-group and, even more, the heavier elements, have been found to be overabundant, contrary to what is expected in $\lambda$ Boo stars (Rachkovskaya 1987).

\section{Conclusions}

We have shown that HD 64491 represents an example of a binary system producing a tangled spectrum that can be easily confused with that of a single star classified as a $\lambda$ Boo star.

The abundance analysis, necessary to confirm its $\lambda$ Boo classification, requires assignment of the correct parameters to each of the system components. This represents an insoluble problem at present; the knowledge of the orbital period and a large set of spectroscopic observations uniformly covering all phases are mandatory for a reliable abundance analysis of this object.

We may recall that among the $\lambda$ Boo candidates there are two known SB2 stars: HD 84948 and HD 171948. The lines of each component are widely separated (see Fig. 1 in Paunzen et al. 1998a), so their spectra can be separated quite easily, but in spite of this a detailed abundance analysis cannot yet be made for lack of sufficient information on the stars' variations.

The situation is much more complicated in the present case of HD 64491; the spectra present broad and shallow wings of the strong metal lines, only slightly displaced with respect to the narrow ones of the primary component on our spectra.

We have demonstrated that the approximate solution of a computed spectrum obtained by the combination of the fluxes of two twin dwarf stars gives rise to the fact that the metal deficiency which would be derived for $\mathrm{Na}$ and $\mathrm{Mg}$ is less pronounced by about 1 dex compared to the abundance computed by considering the star as single.

A metal deficiency of $\mathrm{Na}$ and $\mathrm{Mg}$ of -1.5 dex (i.e. the same that appears in HD 64491) has been computed by Stürenburg (1993) for another $\lambda$ Boo candidate, HD 111786, considered as a single peculiar object. However, we have demonstrated that this star is in fact a multiple system (Faraggiana et al. 2001a).

The real abundances of the two components of HD 64491 are probably not far from the solar ones, but their accurate determination requires the knowledge of further system parameters; the analysis resctricted to a few spectra is not sufficient to arrive at the solution of this intrinsically very complicated problem.

Acknowledgements. We made use of the SIMBAD database operated at the CDS, Strasbourg, France and of the General Catalogue of Photometric Data, available at the Geneva Data Base. We would like to thank the TBL staff members for their assistance, their skilfulness and kindness during these observing runs.

\section{References}

Abt, H. A., \& Morrell, N. 1995, ApJS, 99, 135 
Andrievsky, S. M. 1997, A\&A, 321, 838

Bessell, M. S. 1969, ApJS, 18, 167

Bessell, M. S., Castelli, F., \& Plez, B. 1998, A\&A, 333, 231

Bessell, M. S., Castelli, F., \& Plez, B. 1998, A\&A, 337, 321

Charbonneau, P. 1983, ApJ, 405, 720

De Mey, K., Daems, K., \& Sterken, C. 1998, A\&A, 336, 527

Donati, J.-F., Semel, M., Carter, B. D., Rees, D. E., \& Cameron, A. C. 1997, MNRAS, 291, 658

ESA The Hipparcos Catalogue 1997, ESA SP-1200

Faraggiana, R., \& Bonifacio, P. 1999, A\&A, 349, 521

Faraggiana, R., \& Gerbaldi, M. 1998, Contr. Astron. Obs. Skalnaté Pleso, 27, 413

Faraggiana, R., Gerbaldi, M., Bonifacio, P., \& François, P. 2001a, A\&A, 376, 586

Faraggiana, R., Gerbaldi, M., \& Bonifacio, P. 2001b, A\&A, 380, 286

Gerbaldi, M., Faraggiana, R., Burnage, R., et al. 1999, A\&AS, 137, 273

Gerbaldi, M., Faraggiana, R., \& Balin, N. 2001, A\&A, 379, 162

Gray, R. O. 1997, The Third Conf. on Faint Blue Stars, ed. A. G. Davis Philip, J. W. Liefert, \& R. A. Saffer, 237

Griffin, R., \& Griffin, R. 1986, J. Astrophys. Astr., 7, 195

Griffin, R. E. 2002, AJ, 123, 988

Ilijić, S., Hensberge, H., \& Pavlovski, K. 2001, Lecture Notes in Physics ed. H. M. J. Boffin, D. Steeghs, \& J. Cuypers (SpringerVerlag), 573, 269

Iliev, I. Kh., Paunzen, E., Barzova, I., et al. 2001, IBVS, 5178, 1

Kamp, I., Iliev, I. Kh., Paunzen, E., et al. 2001, A\&A, 375, 899

Kamp, I., Hempel, M., \& Holweger, H. 2002, A\&A, 388, 978

Kazarovets, E. V., Samus, N. N., \& Durlevich, O. V. 2000, The 75th Name-List of Variable Stars, IBVS, 4870, 1
Künzli, M., North, P., Kurucz, R. L., et al. 1997, A\&AS, 122, 51

Kurucz, R. L. 1993, CD-ROM No. 13, 18, Smithsonian Astrophysical Observatory, http://cfaku5.harvard.edu/cdroms.html

Lee, E. K., \& Wright, K. O. 1960, Pub. Dominion Astrophys. Obs. Victoria, 11, 339

Marchetti, E., Faraggiana, R., \& Bonifacio, P. 2001, A\&A, 370, 524

Meynet, G., Mermilliod, J.-C., \& Meader, A. 1993, A\&AS, 98, 477

Moon, T. T. 1985, Comm. from the Univ. of London Obs. 78 and Revisions in 1985, private comm.

Moon, T. T., \& Dworetsky, M. M. 1985, MNRAS, 217, 305

Palmer, D. R. Walker, E. N., Jones, D. H. P., \& Wallis, R. E. 1968, Roy. Obs. Greenwich-Cape Bull., 135, 735

Paunzen, E., Heiter, U., Handler, G., et al. 1998a, A\&A, 329, 155

Paunzen, E., Weiss, W. W., Kuschnig, R., et al. 1998b, A\&A, 335, 533

Rachkovskaya, T. M. 1987, Izv. Krym. Astrofiz. Obs., 76, 3

Rodriguez, E., Lopez-Gonzalez, M. J., \& Lopez de Coca, P. 2000, A\&AS, 144, 469

Schaller, G., Schaerer, D., Meynet, G., \& Maeder, A. 1992, A\&AS, 96, 269

Stürenburg, S. 1993, A\&A, 277, 139

Thompson, G. I., Nandy, K., Jamar, C., et al. 1978, Catalogue of Stellar Ultraviolet Fluxes (The Science Research Council)

Turcotte, S. 2002, ApJ, 573, L129

Uesugi, A., \& Fukuda, I. 1982, Revised Catalogue of Stellar Rotational Velocities, Dept. of Astronomy (Kyoto University)

Venn, K. A., \& Lambert, D. L. 1990, ApJ, 363, 234

Wright, K. O. 1954, Pub. Dominion Astrophys. Obs. Victoria, 10, 1

Wright, K. O., \& Lee, E. K. 1959, Pub. Dominion Astrophys. Obs. Victoria, 11, 59 